\section{R. Wassgren}

\section{E. Brennen \\ Men. ASME.

\author{
M. L. Hunt \\ Mem. ASME.
}

California Institute of Technology, Pasadena, CA 91125

\title{
Vertical Vibration of a
}

Deep Bed of Granular Material in a Container

A deep bed of granular material (more than six layers of particles) was subjected to sinusoidal, vertical vibrations. Several phenomena were observed depending on the amplitude of excitation. These included heaping, surface waves, and arching; the transitions from one state to another involved various dynamic instabilities and bifurcations. The paper includes a description of these phenomena and the characteristic properties associated with each in addition to measurements of the iransitions from one phenomena to another.

\section{Introduction}

A granular material flow typically consists of a particulate solid in an interstitial fluid, usually air, which is subjected to a shearing force. The response of the material is generally quite complicated and is not well understood. To better understand the behavior of these flows, several "benchmark" experiments have been investigated. These include flows in a vertical chute, down an inclined chute, between a fixed wall and moving wall (Couette flow), and in a vibrating container. These benchmark experiments are useful in that they provide a flow environment which is relatively straightforward to model when checking the validity of granular flow theories and simulations. This paper describes a detailed experimental investigation of a container of granular material subjected to vertical oscillation.

In addition to being a useful benchmark experiment, vibration of granular material is of interest for more practical reasons. In industry, vibration is commonly used as a means of handling and transporting particulate materials such as foodstuffs, coal, and pharmaceuticals. Examples include vibrating conveyor belts, hoppers, sorting tables, packing tables, drying plates, and fluidized bed reactors. Vibration of a granular material may also play an important role in natural events such as earthquakes and avalanches. Clearly, understanding how a granular material responds when subjected to vibration can provide valuable design information. More information concerning some of the important issues, questions, and applications of the knowledge of the granular state can be found in a recent review by Jaeger and Nagel (1992).

The standard granular material vibration experiment consists of a granular material such as sand or glass spheres, in a rectangular or cylindrical container mounted on a rigid base which is subjected to vertical, sinusoidal oscillations. This simple experiment exhibits some surprisingly complex behaviors that have yet to be fully explained. These include states in which the particle bed behaves as a "cloud" of particles with little or no structure, and states where the particle bed moves as a coherent mass and exhibits complex wave phenomena. A brief review of these behaviors is given in the following paragraphs.

First, it is important to review some of the variables that will influence the state of the particle bed. These include the

Contributed by the Applied Mechanics Division of THE AMERTAN SOCETY OF MEchanical ENGINEERS for publication in the ASME JoURnal of APPLIED MECHANICS.

Discussion on this paper should be addressed to the Technical Editor, Professor Lewis T. Wheeler, Department of Mechanical Engineering, University of Houston, Houston, TX 77204-4792, and will be accepted until four months after final publication of the paper itself in the ASME JOURNAL OF APPLIED MECHANICS.

Manuscript received by the ASME Applied Mechanics Division, July 18, 1994; final revision, Oct. 23, 1995. Associate Technical Editor: W. N. Sharpe, Jr. amplitude, $a$, and the radian frequency of vibration, $\omega$, the particle properties such as the particle diameter, $d$, and the initial particle bed depth, $h_{0}$. These variables can be combined to form two important nondimensional parameters, the dimensionless bed depth, $h_{0} / d$, which is a measure of the number of particle layers in the bed, and the dimensionless acceleration amplitude, $\Gamma=a \omega^{2} / g$, where $g$ is the acceleration due to gravity. The behavior of the particle bed is strongly related to both of these dimensionless parameters.

For shallow particle beds $\left(h_{0} / d<4\right)$ at dimensionless acceleration amplitudes between $\Gamma=2.5$ and $\Gamma=6.0$, the bed exhibits three states which depend on $h_{0} / d$ (see Thomas et al., 1989 ). In the first, or "Newtonian-I," state ( $h_{0} / d=0.17$ for example) particles bounce around so randomly that the vertical concentration of particles changes very little during a vibration cycle. In the second, or "Newtonian-II," state (for example at $h_{0} / d=0.273$ ) a dense layer of particles accumulates on the vibrating surface once each vibration cycle while the remainder of the particles bounce around randomly. In the third, or "coherent-expanded"' state (for example at $h_{0} / d=1.7$ ), particles begin to move as a coherent mass which expands and contracts considerably during each vibration cycle. When $h_{0} / d$ is increased above 4.0 , the particle bed behavior transitions to what is called a "coherent-condensed," or deep bed state. Here particles move as a coherent mass but remain fairly compact throughout the cycle of vibration. Bachmann (1940) found that the transition from the coherent-expanded state to the coherentcondensed state occurred for large spherical particles when $h_{0} /$ $d \approx 6$. In this deep bed regime, investigators have found that a number of new behaviors appear. These include the formation of heaps, surface waves, and arches.

The "heaping" phenomenon was first observed by Faraday (1831). He found that when sand is placed on a vibrating membrane, small mounds form and a slow bulk convection of particles appears. The same behavior is observed for a granular material in a container performing vertical, sinusoidal oscillations. Above a critical dimensionless acceleration amplitude of $\Gamma=1.2$ (see, for example, Evesque and Rajchenbach, 1989), the free surface of the particle bed begins to form a mound or heap. Generally a single peak is observed to occur either to one side of the container or in the center (Fauve et al., 1989). However, if the vibration level is increased suddenly, multiple peaks sometimes appear (Fauve et al., 1989). The heap is associated with a slow particle convection pattern in which particles avalanche down the free surface of the mound, are subducted into the mass at its lowest point, and then recirculate internally back to the peak.

Although it is perhaps the most extensively studied deepbed vibration phenomenon, the mechanism for heaping and its 
associated convection pattern are not well understood. Several theories explaining the behavior have been offered but none seem to be universally accepted. The reader is referred to papers by Laroche et al. (1989, 1990), Evesque and Rajchenbach (1989), Evesque (1990, 1992), Gallas et al. (1992), and Pak et al. (1995) for further details.

Another phenomenon that is observed for deep beds is the formation of surface waves. Pak and Behringer (1992) (and Behringer, 1993) observed solitary traveling waves that appear during the heaping phenomenon. The waves travel from the lowest point of the heap up the slope to the peak but do not interfere with the continuous avalanche of particles associated with the convection pattern. The waves increase in length and decrease in height as they travel up the slope and eventually disappear at the peak. Though heaping depends only on $\Gamma$, the onset of the traveling surface waves also depends on the vibration-amplitude-to-particle-diameter ratio, $a / d$.

Fauve et al. (1989) and Melo et al. (1994) also observed that when the acceleration level of the external oscillations is increased to the point at which heaping disappears, localized surface waves appear. For certain ranges of the excitation amplitude and frequency, standing surface waves form at half the excitation frequency. Fauve et al. suggest that the formation of these waves occurs because of a resonance between the characteristic particle avalanche time and the vibration period. This mechanism is similar to the Faraday instability (Douady and Fauve, 1988; Miles and Henderson, 1990). Melo et al. studied these waves in three-dimensional containers, as opposed to quasi-two-dimensional containers. They found several different wave patterns, such as stripes and squares, that appear after specific vibrational acceleration amplitudes. They also made measurements of the wavelengths of the standing waves.

Another phenomenon that has been observed in deep beds is known as "arching." This behavior consists of sections of the particle bed oscillating out of phase with one another. The boundaries between these regions are known as "nodes" (called "defects" by Douady et al., 1989) and exhibit very little movement. The resulting motion resembles "collapsing arches." In addition to the bulk oscillating motion of the particle bed, there is a strong convection cell pattern in which particles move down at the nodes and up at the antinodes (the regions between nodes where the peaks and troughs of the arches occur). A more complete description of the individual particle and bed motion is given by Douady et al. (1989). This phenomenon has also been observed in numerical simulations performed by Rosato and Lan (1994).

The arching behavior appears only after the motion of the particle bed undergoes a period doubling bifurcation, which occurs at $\Gamma \approx 4.5$, a value which varies with the depth of the bed (Douady et al., 1989). Prior to the bifurcation, the particle bed has a single flight time that is less than the oscillation period of the base and the bed repeats the same motion every oscillation cycle. After the bifurcation, however, the bed has two flight times: one of which is larger than the oscillation period and one which is less than the oscillation period. Thus the motion of the particle bed is repeated every two cycles instead of every cycle. Since there are two flight times, sections of the bed can be simultaneously undergoing different flight times, producing the arching motion that is observed experimentally ( see Section 3.3 for more detail).

Douady et al. (1989) also found that the number of nodes observed decreases with the height of the bed $\left(h_{0}\right)$. Furthermore, they found that the number of nodes is not determined by the dimensionless acceleration amplitude since different numbers of nodes can be observed for the same value of $\Gamma$. Instead there is a maximum number of nodes that can exist at a particular $\Gamma$ which implies a minimum distance between the nodes. Measurements by Douady et al. indicate that the minimum distance between nodes is proportional to the bed depth, $h_{0}$, and is inversely proportional to the dimensionless acceleration ampli-

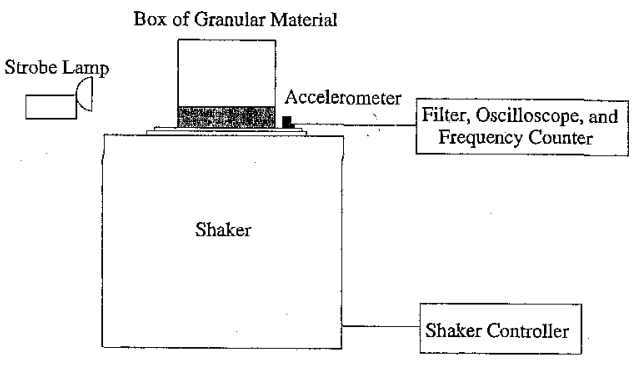

Fig. 1 A schematic of the experimental setup

tude, $\Gamma$. Douady et al. also report that other bifurcations occur at higher acceleration levels.

The present paper presents further studies of the deep-bed phenomena of heaping, surface waves, and arching. Both qualitative and quantitative measurements are reported as well as studies of the transitions between phenomena.

\section{Experimental Apparatus}

Figure 1 shows a schematic of the experimental setup. The apparatus consisted of a box mounted on a Ling electromagnetic shaker (model A-175) which provided sinusoidal, vertical vibrations. The frequency of the oscillations could be controlled to within $\pm 0.5 \mathrm{~Hz}$ while the acceleration level could be controlled to within $\pm 0.1 \mathrm{~g}$. The acceleration of the base of the container was monitored using a Dytran 3126A accelerometer.

Three rectangular lucite boxes were constructed to contain the granular material. The interior vertical sides of the boxes were lined with smooth window glass in order to reduce electrostatic effects. The internal, cross-sectional dimensions were 13.5 $\mathrm{cm}$ by $1.6 \mathrm{~cm}, 18.0 \mathrm{~cm}$ by $1.6 \mathrm{~cm}$, and $18.0 \mathrm{~cm}$ by $4.8 \mathrm{~cm}$. The boxes were mounted on a base of $2.5 \mathrm{~cm}$ thick steel.

Three different sizes of glass spheres (with a density of 2.5 $\mathrm{g} / \mathrm{cm}^{3}$ ) were used. For convenience they are referred to as particles $\mathrm{A}, \mathrm{B}$, and $\mathrm{C}$ and have the following properties:

Particles A: dyed (fluorescent yellow and black) glass spheres with a mean diameter of $1.28 \mathrm{~mm}$ (and a standard deviation of $0.04 \mathrm{~mm}$ ) and a mean sphericity, defined as the largest diameter divided by the smallest diameter, of 1.04 (with a standard deviation of 0.03 ). The angle of repose of the material, $\theta_{r}$, was $30.1 \mathrm{deg}$.

Particles B: 3-mm diameter glass spheres with an angle of repose of $\theta_{r}=31.1 \mathrm{deg}$.

Particles C: 3-mm diameter glass spheres with an angle of repose of $\theta_{r}=26.4 \mathrm{deg}$.

Detailed measurements of particles $B$, and $C$ were not made; however, the diameters of particles $\mathrm{C}$ were more uniform than particles $\mathrm{B}$ and the sphericities of $\mathrm{C}$ were much closer to unity.

All of the experiments were performed with air as the interstitial fluid. In the literature, uncertainty exists as to how important the interstitial fluid is in the formation of the deep-bed phenomena. Recent work by Pak et al. (1995) regarding heap formation indicates that the interstitial fluid is very important for the formation of heaps and the associated convection pattern (although they do mention that other mechanisms such as wall friction or inhomegenous forcing can still produce the observed heap and convection ). They also observed that the interstitial gas effects were most important for deep beds and for small particles (glass spheres, $d<1 \mathrm{~mm}$ ) which is in accord with the studies of interstitial fluid effects by Zeininger and Brennen (1985). In Pak et al.'s experiments with 1-mm diameter glass spheres and $h_{0} / d=100$, the interstitial fluid effects were weak and for $d=$ $1.3 \mathrm{~mm}$ and $h_{0} / d=77$ the effects were not observed at all. Laroche et al. (1989) also found that interstitial fluid effects 
were important for the formation of heaps for particle beds comprised of glass spheres with diameters between 0.63 and $0.8 \mathrm{~mm}$ but they do not mention how beds with larger particles are affected. Evesque (1990), however, reports that in experiments using $0.2,0.4$, and $1-\mathrm{mm}$ diameter glass spheres the interstitial fluid effects were negligible. These experiments did not show any appreciable difference in heap shape or convection pattern for evacuated containers or for containers with mesh walls that allow air to be drawn in from the sides instead of through the particle bed. Despite the disagreement as to the whether or not fluid effects are important for the formation of the bed phenomena for small particles, the experiments performed here used particles and bed depths $\left(d \geq 1.28 \mathrm{~mm}, h_{0} /\right.$ $d<80$ ) that are beyond the regime where Pak et al. regard fluid effects as being important and where Laroche et al. reported their findings. As an additional note recent discrete element simulation work (fluid effects were not included) performed by the authors have reproduced all of the mentioned bed phenomena except for the heaping behavior (Wassgren et al., 1995) further indicating that the phenomena observed here were not the result of interstitial fluid effects.

At a given frequency, $f$, data were obtained for acceleration levels (" $g$ " levels), $\Gamma=a \omega^{2} / g$, between zero and the maximum limit of the shaker (roughly $\Gamma=8.0$ ). For each experiment the acceleration amplitude and frequency were determined from the accelerometer signal. The motion of the particle mass was observed using a strobe lamp while spatial measurements were made using a grid taped to the outside of the rear wall of the box. The flight time of the particle bed (the time between successive collisions) could be measured since collisions between the particle bed and the steel base produced a narrow band of high-frequency noise in the accelerometer output. Thus, by using a high-pass filter, it was possible to measure the time between collisions of the bed with the base.

\section{Observations}

The transition from the coherent-expanded state to the coherent-condensed state was determined by observing when the deep-bed phenomena first appeared as $\Gamma$ was increased. It was found that this transition depended on the dimensionless particle bed depth, $h_{0} / d$, and the type of material. For particles $\mathrm{A}$, the critical $h_{0} / d$ at which the transition occurred was between 5.5 and 8.3. For particles $\mathrm{B}$, the critical $h_{0} / d$ was between 5.0 and 7.5, and for particles $C$ it was between 8.5 and 9.7 .

The deep-bed phenomena observed in the present experiments included heaping, small-amplitude surface waves, arch ing, and large-amplitude surface waves. Two regimes of standing surface waves, defined as small and large-amplitude surface waves, were observed and were similar to the surface waves reported by Fauve et al. (1988) and Melo et al. (1994, 1995). The traveling surface waves described by Pak and Behringer

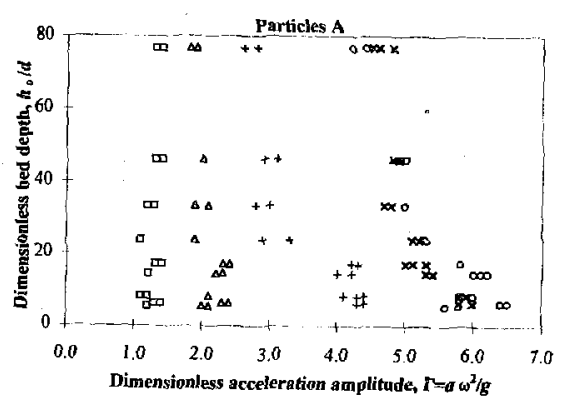

Fig. 2 Incipient values of $\boldsymbol{\Gamma}$ tor the various phenomena as a function of $h_{0} / d_{n}$ Inception values for heaping $\left(\square=\Gamma_{H}\right)$, small-amplitude surface waves $\left(\triangle=\Gamma_{s}\right)$, period doubling bifurcation $\left(+=\Gamma_{B 1}\right)$, return to single flight time $\left(\times=\Gamma_{k_{1}}\right)$, and large-amplitude surface waves $\left(O=\Gamma_{L}\right)$ are shown.

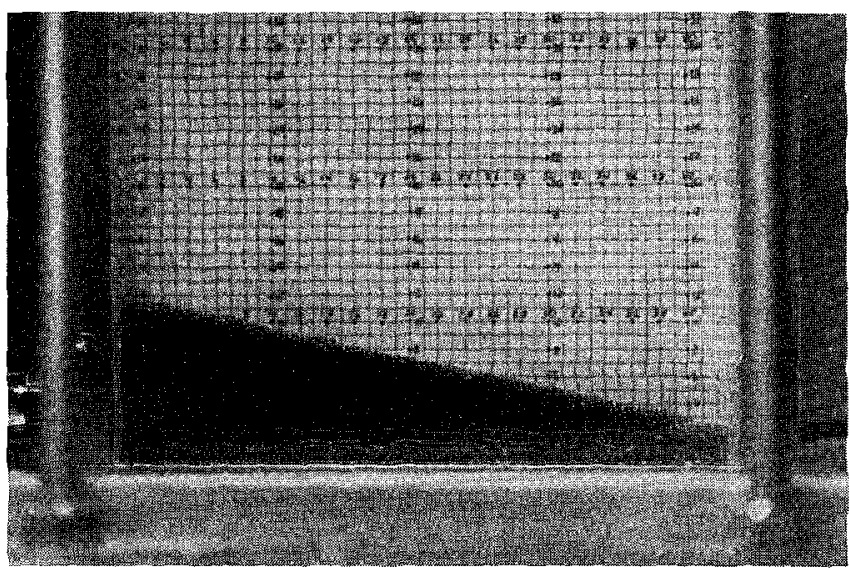

Fig. 3 A photograph of a heap for $\Gamma=1.8, f=30 \mathrm{~Hz}, d=1.28 \mathrm{~mm}$, and $h_{0} / d=17.5$

(1989) were not observed in the present experiments. The sections that follow provide more detailed descriptions of the phenomena.

In addition, measurements were made of the transitions from one phenomenon to, another. These transitions were found to be independent of the both the container size and the excitation frequency, $f$, within the $15 \rightarrow 40 \mathrm{~Hz}$ range, but dependent on the dimensionless acceleration amplitude, $\Gamma$, and the number of particle layers, $h_{0} / d$. This agrees with the results of other experimenters (see, for example, Fauve et al., 1989; Evesque and Rajchenbach, 1989; Melo et al., 1995). Phenomena inception values for particles $A$ are shown in Fig. 2. Although the value at which the transitions between phenomena occurred were different for the three types of particles, the general trends remained the same.

3.1 Heaping. A typical example of a heap is shown in Fig. 3. The inception value for heaping was $\Gamma_{H} \approx 1.2$ and was independent of $h_{0} / d$ and particle type (Fig. 2). These results agree with observations of other experimenters; for example Evesque and Rajchenbach (1989) found that $\Gamma_{H}=1.2 \pm 0.1$. Below the inception value for heaping, the surface of the particle bed remained flat with little particle motion occurring although particles occasionally fell into neighboring voids.

For $\Gamma>\Gamma_{H}$, the initially flat surface of the particle mass started to deform at the side boundaries and the disturbance propagated toward the center of the mass. One side of the particle mass always became thinner while the other side became thicker. The final result was a mound with the peak near a side boundary and a relatively uniform slope across most of the width of the box. Figure 4 indicates the evolution of the heap. For reasons that are obscure, the peak always formed toward the right side of the container for frequencies below $15 \mathrm{~Hz}$ and toward the left side for frequencies above $15 \mathrm{~Hz}$. Multiple peaks and peaks in the center of the box such as described by Fauve et al. (1989) were never observed. Furthermore, when the mound was perturbed with a stirring rod the peak would always reform in its original location. Perhaps, small, unintentional lateral vibrations of the shaker caused this preferential asymmetry (see Evesque, 1992). Asymmetries in the container construction were ruled out by rotating the container $180 \mathrm{deg}$ about a vertical line and observing the that the heap behavior was the same. However, when the container was rotated just $90 \mathrm{deg}$ about a vertical line, the heap showed preferential peaking with a different critical frequency (note that the support structure for the shaker is not radially symmetric). Measurements of the lateral vibrations, for the container mounted in its original location, with an accelerometer indicated maximum accelerations of approximately $0.4 \mathrm{~g}$ at a frequency of $30 \mathrm{~Hz}$ and a vertical acceleration of $1.8 \mathrm{~g}$. For all other vibration parameters the 

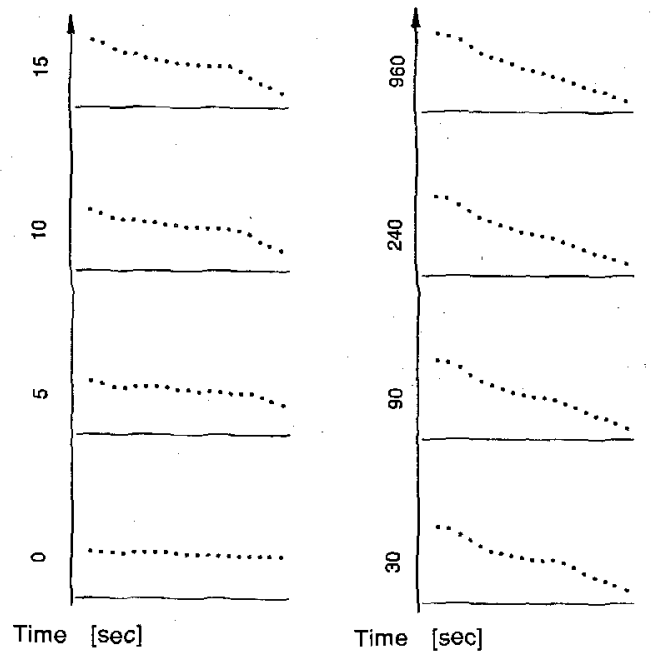

Fig. 4 The evolution of a heap over time. Data for $\Gamma=1.4, f=30 \mathrm{~Hz}$, $d=1.28 \mathrm{~mm}$, and $h_{0} / d=17.5$.

lateral acceleration was smaller than $0.4 \mathrm{~g}$. The maximum final heap slope occurred at this maximum lateral acceleration. To further clarify whether or not these lateral vibrations were affecting the slope of the heap, a discrete element simulation incorporating both vertical and horizontal vibrations was performed. The resulting bed behavior showed a distinct peak to one side of the container and a convection pattern similar to what was observed experimentally.

To document one case (for particles $\mathrm{A}$ and $h_{0} / d=17.5$ ), the height of the particle bed was measured at several locations along the width of the $13.5 \mathrm{~cm}$ by $1.6 \mathrm{~cm}$ box at various times during the evolution of the heap. The mean heap slope at a particular time was determined by a straight line fit through the measured heights at the prescribed locations. Figure 5 presents a typical set of experimental measurements. In all of the runs, the slope increased rapidly at first but then appeared to change much more slowly and approach some final slope. As the acceleration amplitude of the oscillations increased, the final heap slope decreased while the time required to reach the final slope increased.

A distinct convection pattern was always present during the heaping process. Particles avalanched down the slope to its lowest point and were then recirculated within the particle mass back to the peak. Although the peak of the mound was located at one end of the container, it was separated from the wall by a distance of a few particle diameters. Particles next to this boundary moved down along the wall and recirculated back to the peak. The speed of the convective motion appeared to decrease as the slope of the heap decreased implying that the speed of the convective motion decreases with increasing acceleration amplitude.

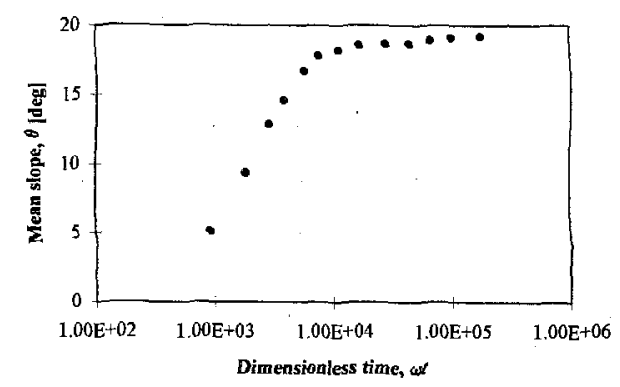

Fig. 5 The mean slope of the heap, $\theta$, as a function of dimensionless time, $\omega t$, at $\Gamma=1.4, f=30 \mathrm{~Hz}, d=1.28 \mathrm{~mm}$, and $h_{0} / d=17.5$
When the motions of the particles during each cycle were studied it was observed that during the time of flight, the particles moved as a single mass with little fluidization. They appeared to be roughly arranged in a hexagonal packing structure and had little translational motion but did rotate in place. When in flight the bottom surface of the particle mass was almost flat but with a slight curvature with the points of least clearance occurring at the walls. Laroche et al. (1989) reported that the smallest clearances occurred beneath the shallowest part of the bed whereas in the present experiments, the smallest clearances occurred beneath the shallowest and deepest parts, both of which were located next to the walls.

3.2 Small-Amplitude Surface Waves. Small-amplitude surface waves began to appear on the slope of the heap when $\Gamma$ was increased above another critical value denoted by $\Gamma_{S} ; \Gamma_{S}$ $\approx 2.2$ but depended on both $h_{0} / d$ and particle type (see Fig. 2). As $\Gamma$ was increased further, the heap and its convection pattern disappeared and the surface waves became more pronounced. Figure 6 is a typical photograph of small-amplitude surface waves.

The small-amplitude waves usually appeared as two-dimensional standing waves with crests parallel to the short dimension of the box although more complicated wave patterns sometimes occurred (for example, sloshing kink waves and cnoidal waves, Guthart, 1992; Miles and Henderson, 1990). Sometimes different portions of the surface would have different standing wave patterns. For example, one end might have waves with crests parallel to the short dimension of the box while the other end might have waves with the crests parallel to the long dimension. When the crests were all parallel to the short dimension, the number of waves varied between $n$ and $n+\frac{1}{2}$ where $n$ is an integer. The walls always coincided with antinodes and the wave motion repeated every two vibration cycles. These characteristics are similar to those found with the parametrically excited Faraday instability waves in liquids (see Miles and Henderson, 1990). Figure 7 shows the wave motion and the trajectories of particles within the waves.

The peaks and troughs of the waves were rounded for small vibration amplitudes, $a$, but the peaks became sharper and the troughs broader as the amplitude increased. In fact as the amplitude increased, the peaks became sharp enough so that particles were ejected from the peaks during the wave motion and would land in the adjacent troughs. Only the top 1-2 layers of particles remained fluidized throughout the wave motion. Below those upper layers, the next 2-4 layers of particles seemed to remain in contact with neighboring particles, but did show movement relative to one another due to the shearing associated with the wave motion. The remaining particles below these upper 3-6

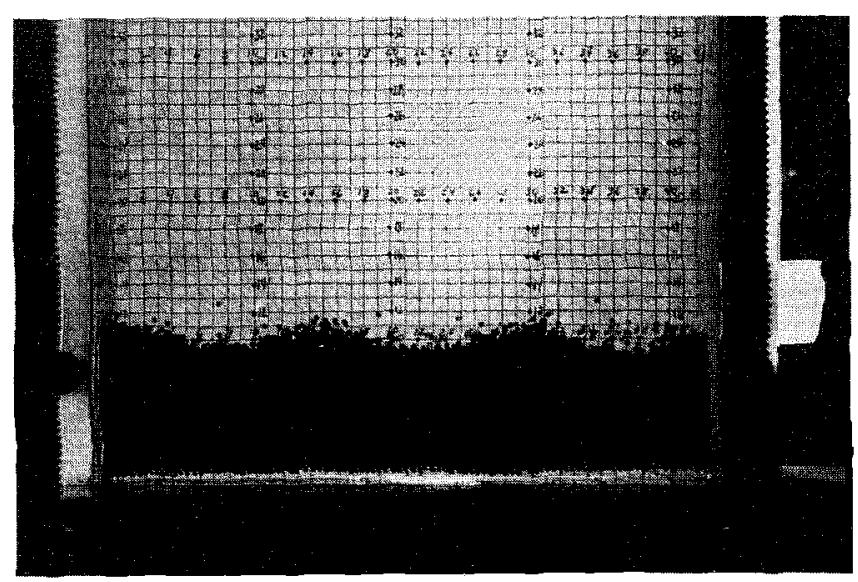

Fig. 6 A photograph of small-amplitude surface waves at $\Gamma=3.3, f=$ $20 \mathrm{~Hz}, d=1.28 \mathrm{~mm}$, and $h_{0} / d=17.5$ 

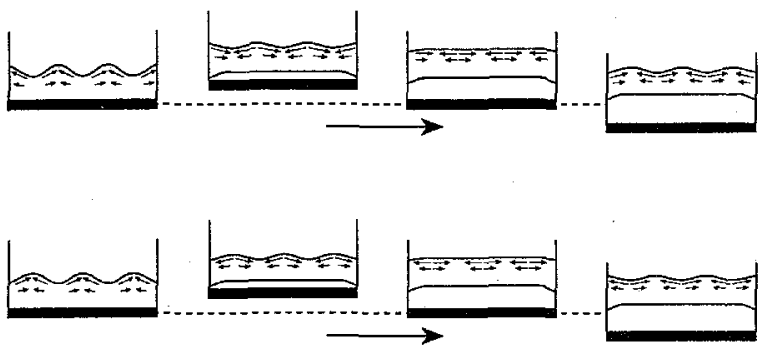

Fig. 7 A schematic showing the motion of the particles for small-amplitude surface waves during two oscillation cycles

layers appeared to remain in a packed state. Note that these observations were made at the vessel walls rather than in the bulk of the material. As $\Gamma$ increased, the fluidized particles in the top few layers became increasingly more active. Although the wave motion was still evident, the fluidized particles "smeared" the waves.

In flight, the bottom surface of the particle mass had a shape similar to that which occurred during heaping; it remained flat with a slight decrease in the clearance near the walls. In the shallow particle beds the waves seemed to disappear near the value of $\Gamma$ at which the flight time of the particle bed bifurcated $\left(\Gamma_{B 1}\right)$. However, in the deeper particle beds they persisted beyond $\Gamma_{B 1}$.

Measurements were made of the wave amplitude, $\eta$ (defined as half of the maximum elevation difference between the peaks and troughs), and the wavelength, $\lambda$, for particles $A$ in the $13.5 \mathrm{~cm}$ by $1.6 \mathrm{~cm}$ box with $h_{0} / d=17.5$. An appropriate dimensionless parameter commonly used in fluid dynamics when investigating surface waves is the Froude number which can be interpreted as the ratio of a characteristic velocity of a flow to the surface wave speed. In Fig. 8 the Froude number based on the wave amplitude, $\eta$,

$$
F r=\frac{a \omega}{\sqrt{g \eta}}
$$

is plotted against $\Gamma$. This figure shows that the wave amplitude correlates well with this Froude number. The Froude number increases with $\Gamma$ starting at $F r=1.0$ and remains greater than 1 throughout the range of $\Gamma$ investigated.

Wavelength measurements were attempted by determining the distance between neighboring wave peaks. This method, however, was not very accurate since the waves peaks were rounded and the precise location of the peak was difficult to determine. Therefore only a few qualitative comments will be made. It appeared that the wave amplitude and wavelength both increased as $h_{0} / d$ increased but then reached a critical value of $h_{0} / d$ where the amplitude and wavelength remained constant.

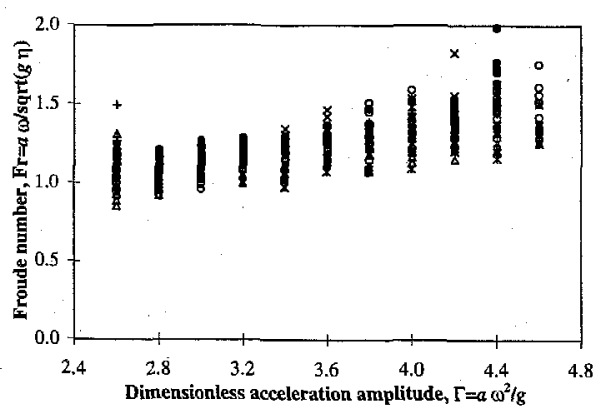

Fig. 8 Froude number, $\mathrm{Fr}=a \omega / \sqrt{g \eta}$, based on the wave amplitude, $\boldsymbol{\eta}$, plotted against the dimensionless acceleration amplitude, $\Gamma$, for smallamplitude surface waves $\left(d=1.28 \mathrm{~mm}\right.$ and $\left.h_{0} / d=17.5\right)$. Frequency key: $+=15 \mathrm{~Hz}, \bullet=20 \mathrm{~Hz}, x=25 \mathrm{~Hz}, O=30 \mathrm{~Hz}, \triangle=35 \mathrm{~Hz}, *=40 \mathrm{~Hz}$.

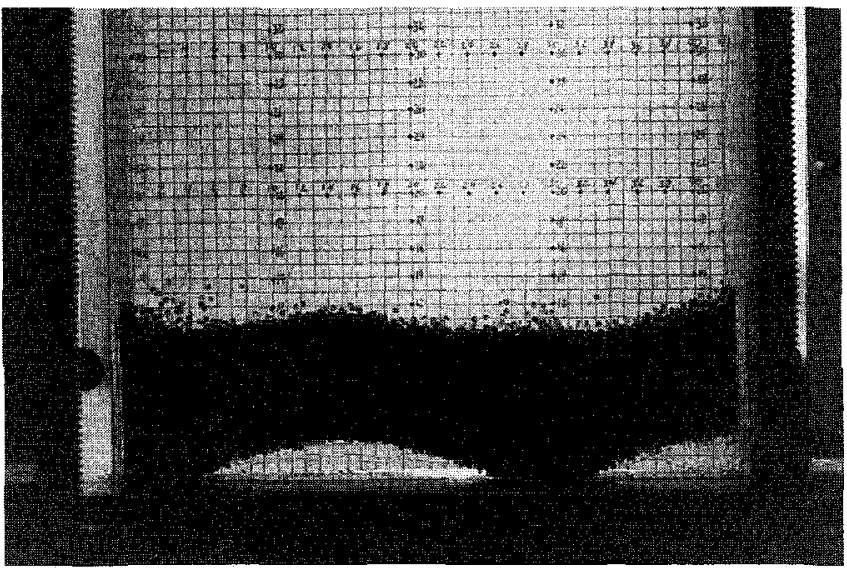

Fig. 9 A photograph of 3-node arching with a single flight time $(\Gamma=8.5$, $f=30 \mathrm{~Hz}, d=1.28 \mathrm{~mm}$, and $h_{0} / d=17.5$ )

This wavelength observation is similar to observations made by Melo et al. (1994) who found that the wavelength increased as $h_{0} / d$ increased but remained constant for values of $h_{0} / d>7$. Other observations indicated that the wavelength increased with increasing particle diameter and was relatively independent of the container width as long as the container width is greater than a wavelength. Both of these observation are consistent with the observations made by Melo et al. (1990).

3.3 Post Bifurcation - Arching. When the acceleration level reached a particular bifurcation value, $\Gamma_{B 1}$, the motion of the particle bed underwent a period doubling bifurcation with respect to the imposed frequency. Instead of repeating the same motion every oscillation cycle, the motion repeated every two cycles. In this regime, sections of the particle bed could oscillate out-of-phase producing the behavior known as arching (see Fig. 9 ). The boundaries between the sections, are termed nodes and oscillated with the smallest amplitude during the motion of the mass. The term antinode is used for the regions between nodes which had the largest amplitudes.

The formation of arches can be visualized by modeling the bed as two completely inelastic particles separated by a fixed horizontal length and vibrating vertically on a sinusoidally oscillating table. Consider first a single inelastic particle. Between collisions with the table, the vertical position, $y$, of the particle follows a ballistic trajectory,

$$
\eta=-\frac{1}{2}\left(\phi-\phi_{n-1}\right)^{2}+\dot{\eta}_{n-1}\left(\phi-\phi_{n-1}\right)+\eta_{n-1},
$$

where the subscript ( $n-1)$ refers to values immediately following the previous collision and the equation is nondimensionalized as follows: $\eta=\omega^{2} y / g, \dot{\eta}=\omega \dot{y} / g$, and $\phi=\omega t$. Collisions between the floor and the particle occur when

$$
\begin{aligned}
-\frac{1}{2}\left(\phi_{n}-\phi_{n-1}\right)^{2}+\dot{\eta}_{n-1}\left(\phi_{n}-\right. & \left.\phi_{n-1}\right) \\
& +\eta_{n-1}-\Gamma \sin \left(\phi_{n}\right)=0
\end{aligned}
$$

where $\phi_{n}$ indicates the dimensionless time of the next collision.

Since the particle is completely inelastic it assumes the velocity of the floor following a collision. If the acceleration of the floor is greater than $-g$ then the particle remains on the floor until the floor acceleration falls below $-g$. By analyzing these equations of motion, one finds that a period doubling bifurcation occurs when $\Gamma_{B 1} \approx 3.7$ (Holmes, 1984; Mehta and Luck, 1990). The subscript $B 1$ refers to the first period-doubling bifurcation. Other period-doubling bifurcations occur at larger $\Gamma$ but only the first will be considered here. 


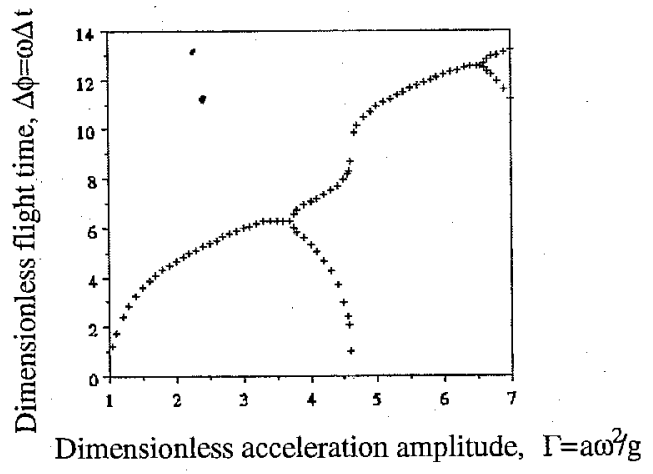

Fig. 10 The dimensionless flight time, $\Delta \phi=\omega \Delta t$ of a completely inelastic particle vibrating on a sinusoidally oscillating table plotted as a function of dimensionless acceleration amplitude, $\Gamma=a \omega^{2} / g$. The first period doubling bifurcation occurs at $\Gamma_{B 1} \approx 3.7$ and the return to a single flight time is at $\Gamma_{B \dagger}^{\star} \approx 4.6$. A second period doubling bifurcation occurs at $\Gamma_{B 2}$ $\approx 6.6$.

Another critical value of $\Gamma$ occurs when $\Gamma_{B 1}^{*} \approx 4.6$. The significance of both $\Gamma_{B 1}$ and $\Gamma_{B 1}^{*}$ becomes apparent by considering two particles on a vibrating table. For $\Gamma<\Gamma_{B 1}$, both particles collide with the floor every oscillation cycle after a flight time of $\Delta t$ which is less than or equal to the period of the forcing oscillations, $T=2 \pi / \omega$ (see Fig. 10). If one of the particles is perturbed, it will return to the same motion it had prior to the perturbation. Thus a line connecting the two particles remains horizontal. For $\Gamma_{B 1}<\Gamma<\Gamma_{B 1}^{*}$ the particles undergo a period-doubling bifurcation and their motion is repeated every two oscillation cycles. Here the particles have two flight times namely $\Delta t_{1}$, which is larger than $T$ and $\Delta t_{2}$ which is less than $T$ (the sum $\Delta t_{1}+\Delta t_{2}$ is less than $2 T$ ). Both particles can either be undergoing the same flight time concurrently (inphase) or can be performing different flight times (out-ofphase). When in-phase, a line connecting the two remains horizontal. On the other hand the out-of-phase motion produces a line that tilts from one side to the other depending on the location of the particles. Thus two different types of motion are exhibited for the same value of $\Gamma$. This same behavior is observed for an entire particle bed. The in-phase motion is known as 0-node arching since no nodes are present and the out-ofphase motion is called $n$-node arching where $n$ is the number of nodes present.

As $\Gamma$ increases, $\Delta t_{1}$ increases and $\Delta t_{2}$ decreases until the next critical value $\Gamma=\Gamma_{B}^{*}$ at which the two flights merge resulting in a single flight time occurring every two cycles. Under these conditions $\left(\Gamma_{B 1}<\Gamma<\Gamma_{B 2}\right.$ where the second period doubling bifurcation occurs at $\Gamma_{B 2} \approx 6.6$ ) only one flight time, $\Delta t_{1}<2 T$, remains but the motion of the particle bed still repeats every two oscillation cycles. Again the particle motions can be either in-phase or out-of-phase.

As an interesting side note, a similar bifurcation also appears for much shallower particle beds (for example $h_{0} / d \approx 0.5$ ) but produces different results. In Brennen et al. (1992) the authors measured the height of a moveable lid containing a shallow bed of particles as a function of $\Gamma$. The height increased suddenly at a critical $\Gamma$. By examining the dynamics of an elastic particle bouncing on a sinusoidally oscillating table (as opposed to the completely inelastic particle just examined), one finds a bifurcation at a particular value of $\Gamma$. In that case the bifurcation caused a "jump" in the maximum height which the particles achieved. Thus, bifurcations occur for both shallow and deep particle beds and can be explained by similar particle bed dynamics by using different effective coefficients of restitution for the collisions between the particles and the base.

Returning to the present observations it was observed that for all three granular materials, the values of $\Gamma_{B 1}$ and $\Gamma_{B 1}^{*}$ decreased as $h_{0} / d$ increased and then reached a constant value

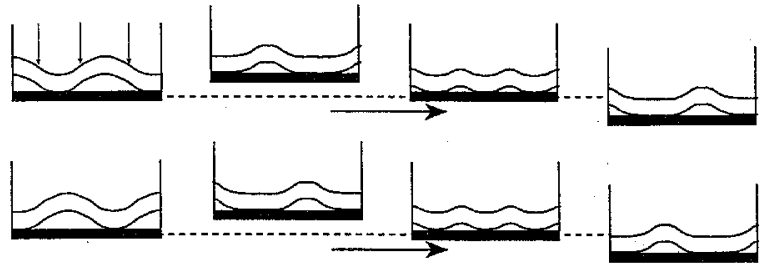

Fig. 11 A schematic showing the particle bed motion for 3-node arching Arrows in the first frame indicate the location of the nodes.

(see Fig. 2). For particles $A, \Gamma_{B 1}$ and $\Gamma_{B 1}^{*}$ decreased from values of 4.2 and 5.8 for $h_{0} / d=5.5$ and reached constant values. of 2.9 and 4.5 for $h_{0} / d \approx 30$. The difference, $\Gamma_{B 1}-\Gamma_{B}^{*}$, remained relatively constant at about 1.6 . Particles $\mathrm{B}$ and $\mathrm{C}$ followed the same trends. The relationship between $\Gamma_{B 1}$ and $h_{0} / d$ is the reverse of that reported by Douady et al. (1989). They found that $\Gamma_{B 1}$ increased with increasing $h_{0} / d$ and suggested that the cause was due to the damping of the interstitial fluid. This discrepancy might be due to the difference in particle sizes used in the experiments. In the experiments by Douady et al., glass spheres with diameters between 0.63 and $0.8 \mathrm{~mm}$ were used (salt was also used), within the regime where interstitial fluid effects are important (Pak et al., 1995). The present experiments, however, used particles with diameters of $1.28 \mathrm{~mm}$ and $3 \mathrm{~mm}$, for which the interstitial fluid effects are negligible.

When $\Gamma_{B 1}<\Gamma<\Gamma_{B 1}^{*}$, either zero, one, or, occasionally, two nodes were present and particles within the mass moved in slow convective cell motions. As $\Gamma$ was increased, the phase difference between the neighboring sections, the amplitude of the arches, and the particle motion within the mass all increased. When $\Gamma_{B 1}^{*}<\Gamma<\Gamma_{B 2}$, neighboring sections of the particle bed oscillated 180 deg out-of-phase. Figure 11 shows the particle bed motion for arching.

At a given $\Gamma$ the number of nodes was not unique. However, a critical $\Gamma$ exists below which a particular number of nodes never occurred. This result is equivalent to the finding by Douady et al. (1989), which indicated the existence of a minimum allowable distance between nodes, $l$. In their experiments using glass spheres with diameters between 0.63 and $0.80 \mathrm{~mm}$, they found $h_{0} / l \approx 0.16(\Gamma-4.2)$. In the present experiments similar measurements were made for particles $\mathrm{A}$ and are presented in Fig. 12 . Clearly $h_{0} / l$ increases with $\Gamma$.

The nodes were positioned symmetrically within the box when three or more nodes were present and the vertical walls always coincided with antinodes. For one or two nodes the arch could be quite asymmetric. In fact, using a stirring rod to perturb the particles, one could change the location of the nodes within the container.

At a given $\Gamma$, the number of nodes could be decreased by perturbing the particle mass. As $\Gamma$ increased, the motion of the

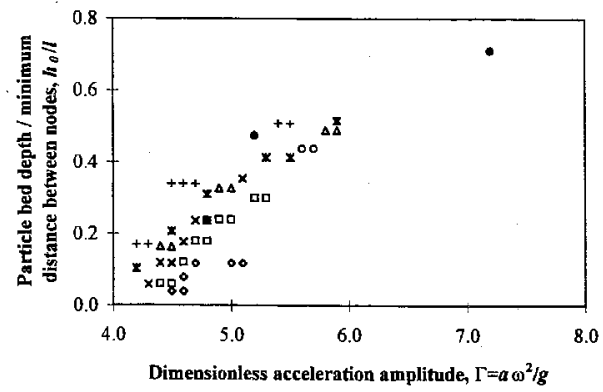

Fig. 12 The particle bed depth, $h_{0}$, divided by the minimum distance between nodes, $I$, as a function of dimensionless acceleration amplitude, $\Gamma$, using particles $A$ with $f=30 \mathrm{~Hz}$ and $d=1.28 \mathrm{~mm}$. Legend: $\diamond=h_{0} / d$ $=5.5, \square=h_{0} / d=6.3, \times=h_{0} / d=8.3, *=h_{0} / d=14.5, \Delta=h_{0} / d=17.2$, $+=h_{0} / d=23.8, \bullet=h_{0} / d=33.3$, and $O=h_{0} / d=46.1$. 


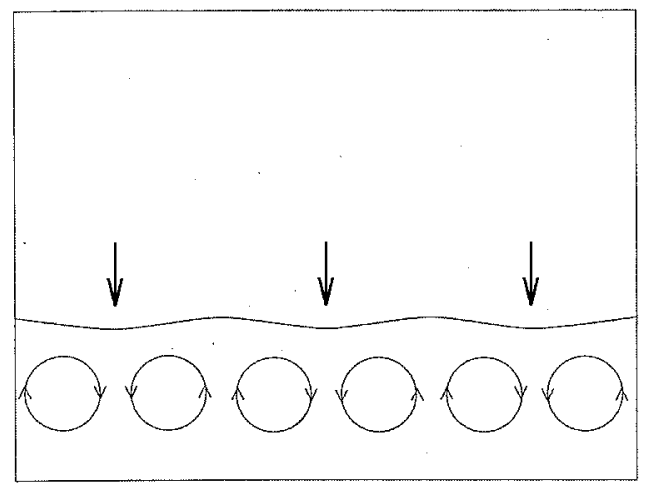

Fig. 13 Particle motion for 3-node arching averaged over several oscillation cycles. The orientation of the convection cells is indicated by the arrows on the circles and the thick arrows indicate the nodes.

particle mass sometimes became erratic, transitioning between 0 -node arching and $n$-node arching ( $n$ is an integer greater than zero). Occasionally the motion settled into arching with a larger number of nodes than before the erratic motion. For shallower beds, the arching behavior with a large number of nodes was more stable than with a small number of nodes. However, as the particle bed became deeper 0 -node arching became the more stable motion.

Particles within the mass moved in circular convection cells when averaged over several oscillation cycles, moving down at the nodes and up at the antinodes (see Fig. 13). The number of convection cells was twice the number of nodes. The particle speed increased with increasing $\Gamma$ and with an increase in the number of nodes but decreased as the frequency increased. The descriptions by Douady et al. (1989) of the particle motions are consistent with the present observations.

For the special case of 0 -node arching, one large convection cell was present similar to the convection pattern observed during heaping. A shallow slope was usually seen beneath a fluidized surface. The undersurface of the particle bed was similar to that observed during heaping; it remained flat with a slight curvature at the walls. Similar experimental observations have been made by Ehrichs et al. (1995); others have observed these features in discrete element simulations (Taguchi, 1992; Gallas et al., 1992; Wassgren et al., 1995). It should be noted that the style of shaking differs somewhat in these various studies. For example, Ehrichs et al. use discrete taps to shake the container instead of continuous, sinusoidal vibration. Moreover, a number of the simulations have fixed walls and a sinusoidally oscillating floor. Convection is observed in all of these studies despite these differences.

3.4 Large-Amplitude Surface Waves. When $\Gamma$ increased above another critical value, $\Gamma_{L}$, large amplitude surface waves appeared superimposed on the arching behavior (see Fig. 14). As shown in Fig. $2, \Gamma_{L}$ occurred very close to the value of $\Gamma_{B 1}^{*}$. As with $\Gamma_{B 1}^{*}, \Gamma_{L}$ decreased with increasing $h_{0} / d$ and eventually reached a constant value near $h_{0} / d \approx 30$.

Large-amplitude waves only formed in regions of the particle bed where the top surface was essentially flat. Thus, waves were seen only during zero, one, and two node arching. For the most part the behavior of the large-amplitude waves was similar to that of the small-amplitude waves (see Section 3.2); however, several differences were apparent. First, the peaks of the large amplitude waves were much sharper than the rounded peaks of the small amplitude waves. Also, the large amplitude waves always formed as standing waves with crests parallel to the short dimension of the container and were large enough that particles were always ejected from the peaks of the waves, landing in the neighboring troughs. Lastly, instead of repeating the same wave motion every two oscillation cycles, the motion was repeated every four cycles.
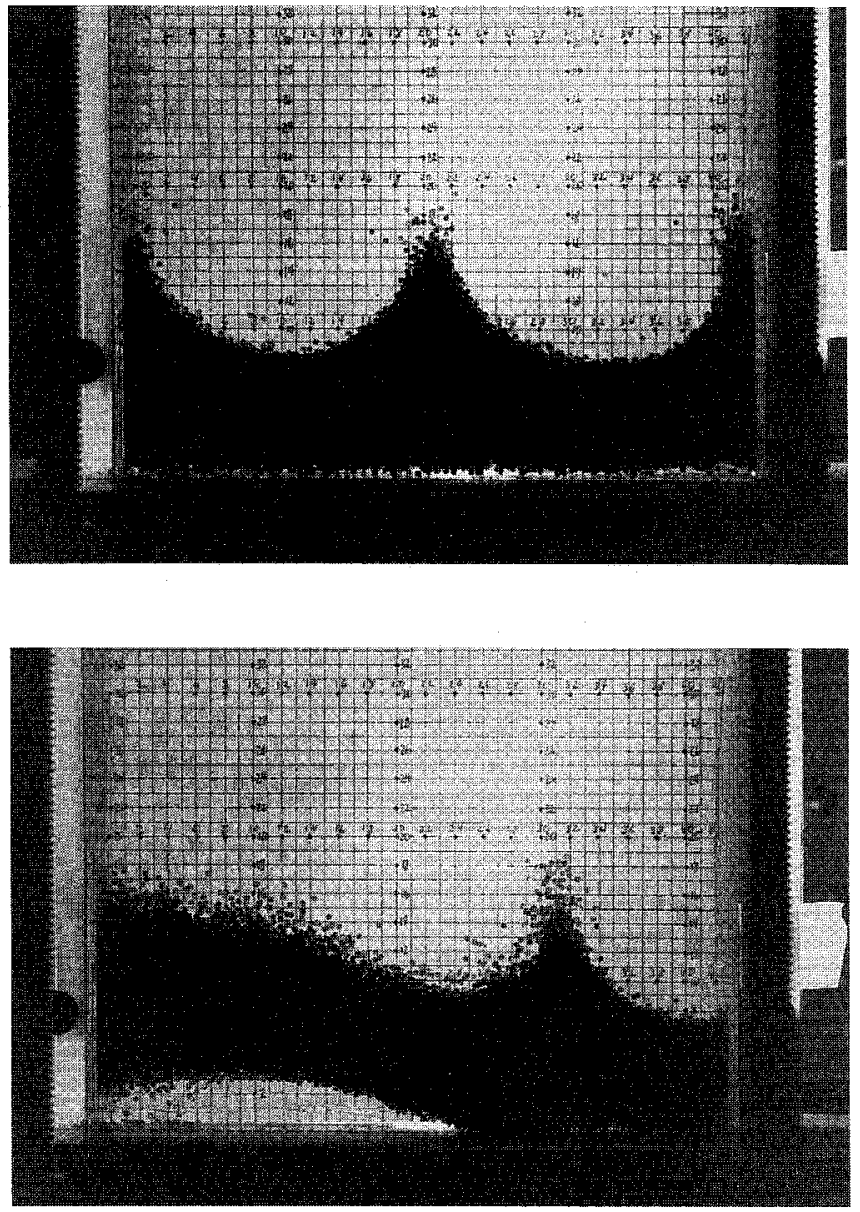

Fig. 14 Two photographs of large-amplitude surface waves. The top photo shows waves superimposed on 0 -node arching and the bottom photo shows waves superimposed on 1-node arching for $\Gamma=6.2, f=20$ $\mathrm{Hz}, d=1.28 \mathrm{~mm}$, and $h_{0} / d=17.5$.
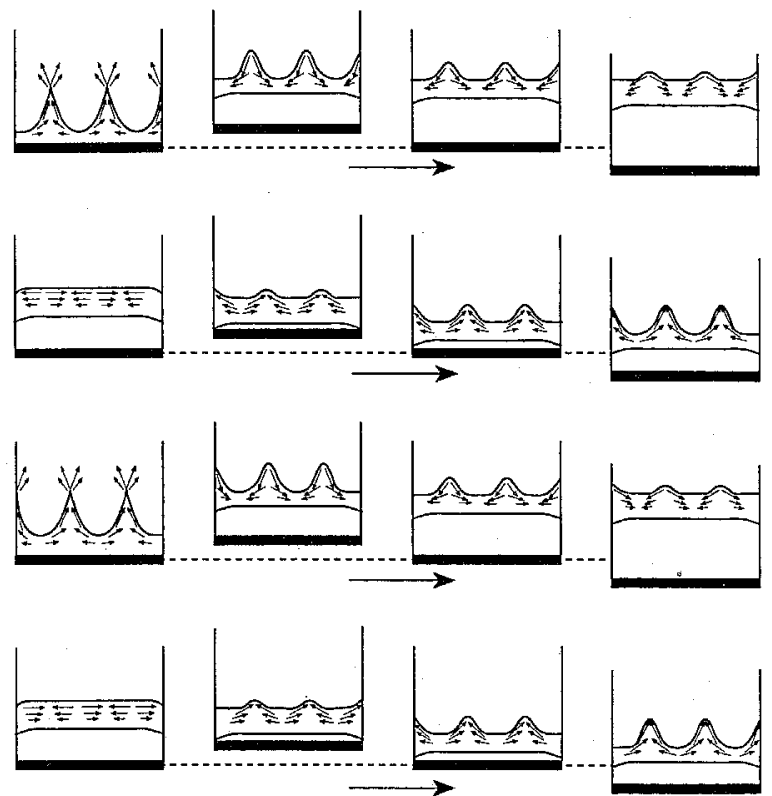

Fig. 15 A schematic showing the motion of the waves and particles for large-amplitude surface waves and 0 -node arching 


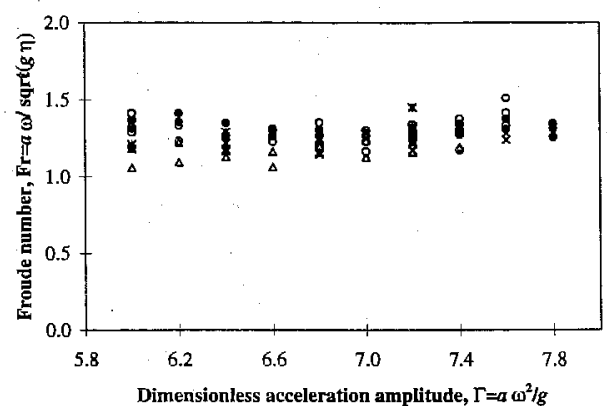

Fig. 16 Froude number, $\mathrm{Fr}=a \omega / \sqrt{g \eta}$, based on the wave amplitude, $\eta$, plotted against the dimensionless acceleration amplitude, $\Gamma$, for largeamplitude surface waves $\left(d=1.28 \mathrm{~mm}\right.$ and $\left.h_{0} / d=17.5\right)$. Frequency key as in Fig. 8.

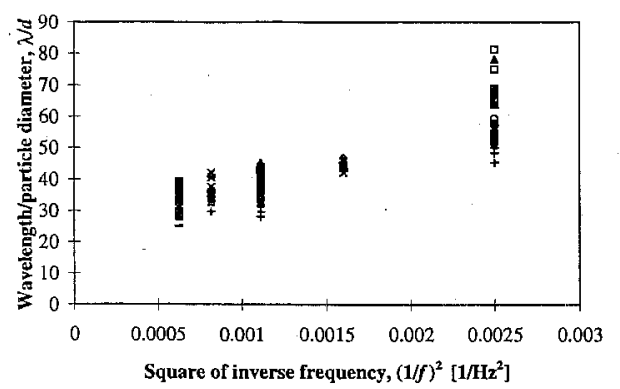

Fig. 17 Wavelength, $\lambda$, plotted against the square of the inverse frequency, $(1 / f)^{2}$, for various dimensionless acceleration amplitudes, $\Gamma$, for large-amplitude surface waves $\left(d=1.28 \mathrm{~mm}\right.$ and $\left.h_{0} / d=17.5\right)$. Dimensionless acceleration amplitude $(\Gamma)$ key: $+=6.0, \bullet=6.2, \times=6.4,0=$ 6.6. $\Delta=6.8, *=7.0,-=7.2, \square=7.4, \forall=7.6$, and $\square=7.8$.

The particle motions within the mass were similar to those observed with small-amplitude waves. Particles at the surface were fluidized while within the mass they remained in a packed state but moved relative to one another due to the shearing of the wave motion which was much greater than for small-amplitude waves. The number of waves present was given by $n / 2$ and the lateral boundaries always coincided with antinodes. Figure 15 shows a schematic of the wave motion with 0 -node arching.

Measurements were made of the wave amplitude, $\eta$, and the wavelength, $\lambda$, for particles $A$ in the $13.5 \mathrm{~cm}$ by $1.6 \mathrm{~cm}$ box with $h_{0} / d=17.5$. Again the amplitude data correlates well with the Froude number of Eq. (1); Fig. 16 shows that Fr is close to constant at a value of about 1.2 ; note that this is the same as for the small-amplitude waves.

Wavelength measurements were made by measuring the distance between peaks and are more accurate than the small-amplitude data since the peaks are well defined and concave. The wavelengths for various acceleration amplitudes are plotted against the square of the inverse frequency in Fig. 17 in order to compare with the measurements made by Melo et al. (1994). Clearly the wavelength data correlates well with the square of the inverse frequency, as was the case with the data of Melo et al.

As with the small-amplitude waves (see Section 3.2), both the amplitude and wavelength appear to increase with increasing $h_{0} / d$ but then saturate at a critical value of $h_{0} / d$. Additionally, as $\Gamma$ is increased, the waves begin to "tilt" alternately from side to side with neighboring peaks either pointing toward or away from one another.

\section{Conclusions}

When a vessel containing a deep bed of granular material is vibrated, a wide range of different flow phenomena can occur depending on the level of the imposed vibration. These phenomena are heaping, surface waves, and arching. Each of these involves complex two and three-dimensional particle motions. Many of the details of the particle motions remain to be understood. This paper provides some quantitative documentation of these complex dynamical phenomena and, in some cases, a tentative framework for an understanding of the bifurcations that occur in the dynamics.

\section{Acknowledgments}

The authors wish to acknowledge the support of the National Science Foundation who supported this work under NSF grant CTS 9300665.

\section{References}

Bachmann, D., 1940, Verfahrenstechnik Z.V.D.I. Beiheft, No. 2, p. 43, (cited by Gutman, 1976a)

Behringer, R., 1993, "The dynamics of flowing sand," Nonlinear Science Today, Vol. 3, No. 3, pp. 1-15.

Brennen, C., Ghosh, S., and Wassgren, C., 1993, "Vertical Oscillation of a Bed of Granular Material," ASME JouRNaL of APPLIEd MECHANICS, Vol. 63, pp. $156-161$.

Douady, S., and Fauve, S., 1988, "Pattern selection in Faraday instability," Europhys. Lett., Vol. 6, No. 3, pp. 221-226.

Douady, S., Fauve, S., and Laroche, C., 1989, "Subharmonic instabilities and defects in a granular layer under vertical vibrations," Europhys. Lett., Vol. 8, No. 7, pp. $621-627$

Ehrichs, E., Jaeger, H., Karczmar, G., Knight, J., and Kuperman, V., 1995, "Granular convection observed by magnetic resonance imaging," Science, Vol. 267, No. 5204, pp. $1632-1634$.

Evesque, P., 1990, "Comment on: Convective flow of granular masses under vertical vibrations," J. Phys. France, Vol. 51, No. 8, pp. 697-699.

Evesque, P., 1992, "Shaking dry powders and grains,"' Contemp. Phys., Vol. 33, No. 4, pp. $245-261$.

Evesque, P., and Rajchenbach, J., 1989, "Instability in a sand heap," Phys. Rev. Lett., Vol. 62, No. 1, pp. 44-46.

Faraday, M., 1831, "On a peculiar class of acoustical figures and on certain forms assumed by groups of particles upon vibrating elastic surfaces," Phil. Trans. R. Soc. London, Vol. 52, pp. 299-340.

Fauve, S., Douady, S., and Laroche, C., 1989, "Collective behaviors of granula masses under vertical vibration," J. Phys. France, Vol. 50, No. 3, pp. 187-191.

Gallas, J., Herrmann, H., and Sokolowski, S., 1992, "Convection cells in vibrating granular media," Phys. Rev. Lett.; Vol. 69, No. 9, pp. 1371-1374.

Guthart, G., 1992, "On the existence and stability of standing solitary waves in Faraday resonance," Ph.D. Thesis, California Institute of Technology, Pasadena, CA.

Holmes, P., 1982, "The dynamics of repeated impacts with a sinusoidally vibrating table," $J$. of Sound and Vibration, Vol. 84, No. 2, pp. 173-189.

Jaeger, H., and Nagel, S., 1992, "Physics of the granular state," Science, Vol: 255, No. 5051, pp. 1523-1531.

Laroche, C., Douady, S, and Fauve, S., 1990, "Convective flow of granular masses under vertical vibrations," J. Phys. France, Vol. 50, No. 7, pp. 699-706. Laroche, C., Douady, S., and Fauve, S., 1990, "Reply to Comment on: Convective flow of granular masses under vertical vibrations," J. Phys. France, Vol. 51, No. 8 , p. 700 .

Mehta, A., and Luck, J., 1990, "Novel temporal behavior of a nonlinear dynamical systein: The completely inelastic bouncing ball," Phys. Rev. Lett., Vol. 65, No. 4 , pp. $393-396$

Melo, F., Umbanhowar, P., and Swinney, H., 1994, "Transition to parametric wave patterns in a vertically oscillated granular layer,' Phys. Rev. Lett., Vol. 72, No. 1 , pp. $172-175$

Melo, F., Umbanhowar, P., and Swinney, H., 1995, "Hexagons, kinks, and disorder in oscillated granular layers," Phys. Rev. Lett., Vol. 75, No. 21, pp. 3838-3841.

Miles, J., and Henderson, D., 1990, "Parametrically forced surface waves," Annual Rev. Fluid Mech., Vol. 22, pp. 143-165.

Pak, H., and Behringer, R., 1993, "Surface waves in vertically vibrated granula materials," Phys Rev. Lett. Vol. 71, No. 12, pp. 1832-1835.

Pak, H., Van Doorn, E., and Behringer, R., 1995, "Effects of ambient gases on granular materials under vertical vibration,"' Phys. Rev. Lett., Vol. 74, No. 23 , pp. 4643-4646.

Rosato, A., and Lan, Y., 1994, "Granular dynamics modeling of vibrationinduced convection of rough inelastic spheres,' Proceedings of the First Interna tional Particle Technology Forum, Denver, CO.

Thomas, B., Mason; M., Liu, Y., and Squires, A., 1989, "1dentifying states in shallow vibrated beds,”' Powder Tech., Vol. 57, pp. 267-280.

Wassgren, C. R., Brennen, C. E., and Hunt, M. L., 1995, "Discrete element simulations of a deep bed of granular material subjected to vertical, sinusoida vibrations," manuscript in preparation.

Zeininger, G., and Brennen, C. E., 1985, "Interstitial fluid effects in hoppe flows of granular material," ASME Cavitation and Multiphase Flow Forum, Vol 23, pp. 132-136. 